\title{
Spin qubits with electrically gated polyoxometalate molecules
}

\author{
Jörg Lehmann, ${ }^{1}$ Alejandro Gaita-Ariño, ${ }^{1,2}$ Eugenio Coronado, ${ }^{2}$ and Daniel Loss ${ }^{1}$ \\ ${ }^{1}$ Department of Physics und Astronomy, University of Basel, \\ Klingelbergstrasse 82, CH-4056 Basel, Switzerland \\ 2 Instituto de Ciencia Molecular, Universitat de Valencia, \\ Polígono de La Coma, s/n, E-46980 Paterna, Spain
}

(Dated: November 5, 2018)

\begin{abstract}
Spin qubits offer one of the most promising routes to the implementation of quantum computers. Very recent results in semiconductor quantum dots show that electrically-controlled gating schemes are particularly well-suited for the realization of a universal set of quantum logical gates. Scalability to a larger number of qubits, however, remains an issue for such semiconductor quantum dots. In contrast, a chemical bottom-up approach allows one to produce identical units in which localized spins represent the qubits. Molecular magnetism has produced a wide range of systems with tailored properties, but molecules permitting electrical gating have been lacking. Here we propose to use the polyoxometalate $\left[\mathrm{PMo}_{12} \mathrm{O}_{40}(\mathrm{VO})_{2}\right]^{q-}$, where two localized spins-1/2 can be coupled through the electrons of the central core. Via electrical manipulation of the molecular redox potential, the charge of the core can be changed. With this setup, two-qubit gates and qubit readout can be implemented.
\end{abstract}

\section{INTRODUCTION}

Quantum dots have often been termed artificial atoms and molecules. $\frac{1}{-}$ Indeed, their tunability via electrical gates has permitted one to reach into a regime where only a single electron sits in each dot. A double dot then becomes the analogue of a hydrogen molecule. The Heisenberg exchange coupling in such a system can be manipulated by appropriately chosen electrical pulse sequences, up to a point which has not yet been achieved for its real counterpart. 2 Recently, it has been demonstrated that this technique allows the realization of the fundamental one- and two-qubit quantum gates ${ }^{3,4,5}$ in such a system. 6.7

Naturally, the question arises whether the successful schemes and techniques of an electrical control of spins in quantum dots can be transfered back to electron spins in single molecules. In view of the recent experimental progress in the field of molecular electronics, which demonstrates that magnetic transitions in atomic chains 8 and single molecules ${ }^{9.10}$ can be resolved in transport measurements, such a goal should in principle be achievable. In particular, the field of molecular magnetism ${ }^{11,12}$ has provided a plethora of systems of almost arbitrary magnetic functionality. $13,14,15,16,17$ Very recently, phasecoherence times of up to $3 \mu \mathrm{s}$ have been reported for such molecular nanomagnets after deuteration. 18 Yet, a suitable system for an electrical control of molecular qubits has still been missing. It is this electrical control which is crucial for scalability and which distinguishes this proposal from earlier ones also based on molecular magnets 19,20

Here we propose an experimental setup which permits the electrical switching of the exchange interactions between two electron spins. The system we choose for illustrating our proposal is $\left[\mathrm{PMo}_{12} \mathrm{O}_{40}(\mathrm{VO})_{2}\right]^{q-}, 21$ a polyoxometalate ${ }^{17}$ which consists of a central mixed-valence core based on the $\left[\mathrm{PMo}_{12} \mathrm{O}_{40}\right] \mathrm{Keggin}^{22}$ unit, able to act as an electron reservoir accommodating a variable number of delocalized electrons hopping over the Mo centers, capped by two vanadyl groups containing two localized spins (cf. Fig. 1) The spins on these two $(\mathrm{VO})^{2+}$ units (a)

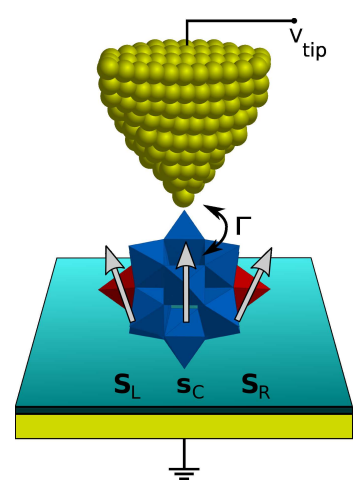

(b)

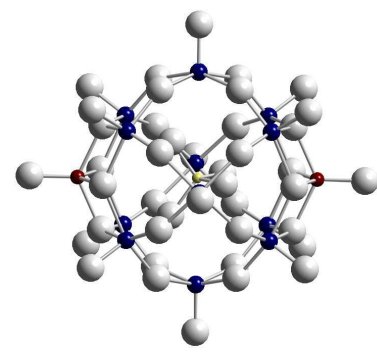

FIG. 1: (a) Schematic drawing of the polyoxometalate $\left[\mathrm{PMo}_{12} \mathrm{O}_{40}(\mathrm{VO})_{2}\right]^{q-}$ separated by a tunnelling barrier from a metallic substrate and contacted via a tunnel coupling $\Gamma$ to a tip at a potential $V_{\text {tip }}$. Indicated are the two localized spins $\mathbf{S}_{\mathrm{L}}$ and $\mathbf{S}_{\mathrm{R}}$ of the $\mathrm{V}$ atoms in the center of the red square pyramids. Depending on the redox state of the molecule, the delocalized valence electrons of the Mo atoms in the center of the blue octahedra form a spin $\mathbf{S}_{\mathrm{C}}$ or pair to a singlet. The $\mathrm{O}$ anions are located at the vertices of the polyhedra. (b) Ball-and-stick model of the polyoxometalate: O (grey), Mo (blue), V (red), and P (yellow).

are weakly magnetically coupled via the delocalized electrons of the central core. We shall show how this magnetic coupling can be switched in an all-electrical way and how this can be used for the implementation of a fundamental two-qubit gate (providing entanglement), the socalled square-root-of-swap $\sqrt{\mathrm{SWAP}} \underline{\underline{4}}$ Furthermore, we will detail how to use a variation of this procedure for the readout of the final state of the two qubits. 


\section{LOW-ENERGY MODEL OF THE POLYOXOMETALATE}

For the description of the low-energy states of the polyoxometalate, two cases have to be distinguished: For an even number of electrons on the mixed-valence Keggin core, their spins pair antiferromagnetically to form a total spin 0 state. Then the system can be modelled by the two spins $1 / 2$ on the vanadyl groups weakly coupled via an indirect exchange mechanism mediated by the core electrons. On the other hand, if the number of core electrons is odd, an unpaired spin $1 / 2$ on the core remains and one obtains a set of three coupled spins $1 / 2$. Restricting ourselves to two charge states differing by one electron, we can choose the electron number of the even state as reference and write the Hamiltonian in the form

$$
\begin{aligned}
H= & -J\left(n_{\mathrm{C}}\right) \mathbf{S}_{\mathrm{L}} \cdot \mathbf{S}_{\mathrm{R}}-J_{\mathrm{C}}\left(\mathbf{S}_{\mathrm{L}}+\mathbf{S}_{\mathrm{R}}\right) \cdot \mathbf{s}_{\mathrm{C}} \\
& +\left(\epsilon_{0}-e V_{\mathrm{g}}\right) n_{\mathrm{C}}+U n_{\mathrm{C}}\left(n_{\mathrm{C}}-1\right) / 2 .
\end{aligned}
$$

Here, the first term describes the indirect-exchange coupling between the left and right spins $\mathbf{S}_{\mathrm{L}}$ and $\mathbf{S}_{\mathrm{R}}$ with a coupling constant $J\left(n_{\mathrm{C}}\right)$ which depends on the number of electrons $n_{\mathrm{C}}$ - measured with respect to the reference number - on the central core of the molecule. In the case of an electron being localized on the core, its spin $\mathbf{s}_{\mathrm{C}}=(1 / 2) \sum_{\sigma \sigma^{\prime}} d_{\mathrm{C} \sigma}^{\dagger} \boldsymbol{\tau}_{\sigma \sigma^{\prime}} d_{\mathrm{C} \sigma^{\prime}}$ couples to the left and right spins with coupling constant $J_{\mathrm{C}}$. Here, the operators $d_{\mathrm{C}, \sigma}\left(d_{\mathrm{C}, \sigma}^{\dagger}\right)$ destroy (create) and electron on the central core and $\boldsymbol{\tau}$ is the vector of the three Pauli matrices. The two last terms contain the orbital energy $\epsilon_{0}$ of the electron on the central core, which can be shifted due to a gate potential $V_{\mathrm{g}}$, and the molecule's charging energy $U$, respectively. The latter is assumed to be the largest energy scale of the problem. We consider a situation where the central core of the molecule is tunnelcoupled to one or more metallic leads $\ell$ permitting electrons to flow on and off the molecule with tunnelling rates $\Gamma_{\ell}$. In the STM setup depicted in Fig.1(a), two leads, the tip and the metallic surface, are present. We assume that an insulating layer-which still allows electrons to tunnel through - between the surface and the molecule leads to a molecule-surface coupling which is much smaller than the typical energy scale $J_{\mathrm{C}}$ of the molecular Hamiltonian (1). An applied bias voltage $V_{\mathrm{b}}=V_{\text {tip }}$ then leads to a shift of the molecular levels which to a very good approximation is described by the linear relation $V_{\mathrm{g}}=\eta V_{\text {tip }}$ with $0<\eta<1.23$ Note that in a more sophisticated three-terminal setup, the parameters $V_{\mathrm{g}}$ and $V_{\mathrm{b}}$ can be controlled independently. ${ }^{24,25}$

\section{IDEAL QUANTUM-GATE OPERATION}

The $\sqrt{\text { SWAP }}$ operation is defined by $|\chi, \lambda\rangle \rightarrow$ $(|\chi, \lambda\rangle+\mathrm{i}|\lambda, \chi\rangle) /(1+\mathrm{i})$, where $\chi, \lambda=\uparrow, \downarrow$. For electrically confined quantum dots, it can be physically implemented by an appropriately chosen gate-voltage pulse which turns on the Heisenberg exchange coupling between the qubits for a specific time (see below). $\underline{\underline{4}}$ In a molecular system, however, the exchange constants are fixed by the chemical structure and cannot be directly changed. Thus, a more sophisticated quantumgate scheme has to be employed. We propose to indirectly manipulate the coupling between the two qubits by changing the occupation $n_{\mathrm{C}}$ of the central core in the molecule described above. This change in $n_{\mathrm{C}}$ can be induced by changing the gate voltage $V_{\mathrm{g}}$, such that different charge sectors of the molecule become stable (cf. Fig. 2). Chemically this amounts to a change in the redox potential of the molecule. We will first discuss the basic idea of the quantum-gate scheme, thereby assuming that the electron number $n_{\mathrm{C}}$ can be changed in a deterministic, externally controllable way. Later on, this assumption will be relaxed and the full tunnelling dynamics due to the change in $V_{\mathrm{g}}$ will be included.

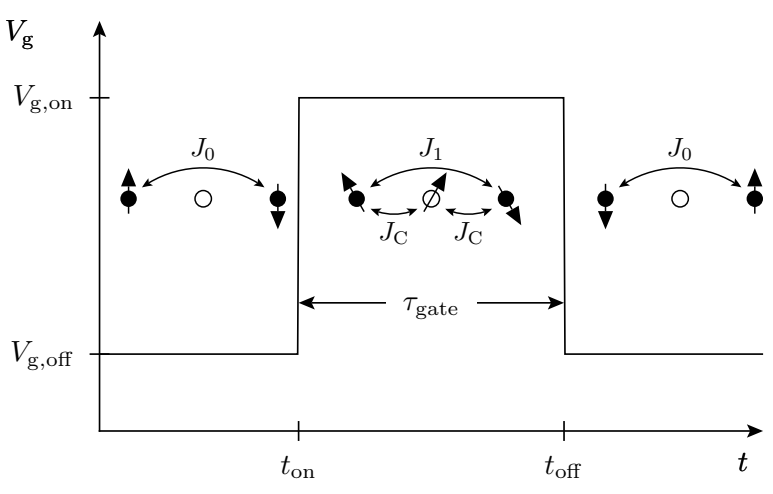

FIG. 2: Quantum-gate sequence for the $\sqrt{\text { SWAP }}$ operation and exchange coupling constants during the corresponding gate phase. The gate is turned on for a time $\tau_{\text {gate }}$.

Initially, one adjusts the gate voltage $V_{\mathrm{g}}$ in such a way that the stable configuration is given by an even number of electrons on the central core $\left(n_{\mathrm{C}}=0\right)$. We assume that the exchange coupling $J_{0}=J(0)$ between the spins $\mathbf{S}_{\mathrm{L}}$ and $\mathbf{S}_{\mathrm{R}}$ will then be very small and can be disregarded for times much smaller than $\hbar / J_{0}$ (see also below). The quantum-gate operation begins by changing the gate voltage such that the $n_{\mathrm{C}}=1$ charge sector becomes stable. Then, on a time-scale of the inverse tunnelling rate, an electron enters the central core. After this has happened, the dynamics of the three-qubit system is governed by the Hamiltonian (1) in the $n_{\mathrm{C}}=1$ subspace. The spin-dependent part of this Hamiltonian becomes

$$
H_{1}=-\left(J_{1}-J_{\mathrm{C}}\right) \mathbf{S}_{\mathrm{L}} \cdot \mathbf{S}_{\mathrm{R}}-\frac{J_{\mathrm{C}}}{2} \mathbf{S}^{2},
$$

where $\mathbf{S}=\mathbf{S}_{\mathrm{L}}+\mathbf{S}_{\mathrm{R}}+\mathbf{s}_{\mathrm{C}}$ is the total spin of the system and $J_{1}=J(1)$. Before considering the time-evolution due to this Hamiltonian, we conclude our discussion of the gate cycle: after a specific time $\tau_{\text {gate }}$, one switches back to the initial gate voltage and the excess electron tunnels off the central core again. After this has happened, the 
two outer spins are, up to times much smaller than $\hbar / J_{0}$, decoupled again.

The only non-trivial dynamics is induced by the Hamiltonian (2). The first term contains an effective exchange coupling between the two spins. Up to an irrelevant phase factor, its corresponding time-evolution yields the $\sqrt{\text { SWAP }}$ gate if the gate time $\tau_{\text {gate }}$ fulfils 4

$$
\left(J_{1}-J_{\mathrm{C}}\right) \frac{\tau_{\text {gate }}}{\hbar}=\frac{\pi}{2}+2 \pi n,
$$

with $n$ being an arbitrary integer. The second term in equation (2) depends on the total spin of the three-spin$1 / 2$ system. While this quantity is unknown, we can eliminate its influence on the gate behavior if we restrict ourselves to stroboscopic gate times $\tau_{\text {gate }}$ for which the contribution $\left.\exp \left[\mathrm{i}\left(J_{\mathrm{C}} / 2\right) \mathbf{S}^{2} \tau_{\text {gate }} / \hbar\right)\right]$ to the time-evolution operator is trivial. Evaluating the effect of the operator $\mathbf{S}^{2}$ in the eigenbasis (see also equation (6) below), this is the case for gate times given by

$$
\tau_{\text {gate }}=\frac{4 \pi}{3} \frac{\hbar}{\left|J_{\mathrm{C}}\right|} m
$$

with the arbitrary integer $m>0$. It is important to note that relation (4) is independent of the spin direction of the additional electron. Equating conditions (4) and (3), we obtain the following relation between $J_{1}$ and $J_{\mathrm{C}}$ :

$$
\frac{J_{1}}{\left|J_{\mathrm{C}}\right|}=\operatorname{sgn} J_{\mathrm{C}}+\frac{3}{8} \frac{1-4 n}{m} \text {. }
$$

\section{REALISTIC QUANTUM-GATE BEHAVIOR}

In the preceding discussion we have assumed that the tunnelling process of the electron is highly controlled, i.e., that we are able to instantaneously switch between the $n_{\mathrm{C}}=0$ and $n_{\mathrm{C}}=1$ states. In reality, tunnelling is a probabilistic event occurring on a mean time scale of the order of the inverse tunnelling rate. In order to investigate to what extent this stochasticity affects our analytical findings, we compare them with numerical results obtained from a simulation of the incoherent quantum dynamics using the average gate fidelity $\mathcal{F}$ as figure of merit for the gate process (see App. A ). In Fig. 3, we show this quantity as a function of the gate time and the ratio $J_{1} /\left|J_{\mathrm{C}}\right|$ for the case of a ferromagnetic coupling $J_{\mathrm{C}}>0$ (the antiferromagnetic case behaves very similarly). We focus on the strong-coupling regime $\hbar \Gamma \gg\left|J_{\mathrm{C}}\right|$, where tunnelling proceeds rapidly compared to the internal coherent dynamics. As expected from our analytical considerations, the average gate fidelity assumes maxima whenever both conditions (3) and (4) are fulfilled. We found the gate error $1-\mathcal{F}$ to decrease with increasing tunnelling rate $\Gamma$. For $\Gamma=6\left|J_{\mathrm{C}}\right| / \hbar$, for instance, we obtain a fidelity $\mathcal{F}=0.99$ at the maximum indicated by the circle in Fig. 3.

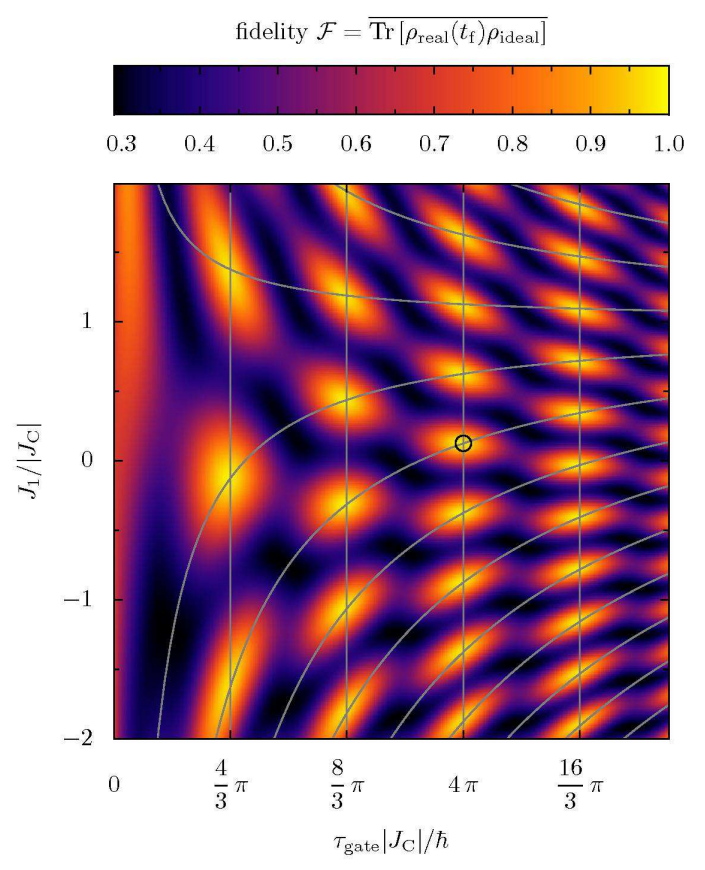

FIG. 3: Average gate fidelity $\mathcal{F}$ as a function of the gate time $\tau_{\text {gate }}$ and the ratio $J_{1} /\left|J_{\mathrm{C}}\right|$ for a ferromagnetic $J_{\mathrm{C}}>0$. The conditions (3) and (4) are indicated by solid lines. The parameters are: $\hbar \Gamma=5\left|J_{\mathrm{C}}\right|, k_{\mathrm{B}} T=0.001\left|J_{\mathrm{C}}\right|$, and $V_{\mathrm{g}, \text { on }}=-V_{\mathrm{g}, \text { off }}=$ $15\left|J_{\mathrm{C}}\right|$ (measured from the charge-degeneracy point).

\section{READOUT PROCESS}

We now show that in a molecular system described by the Hamiltonian (1), it is possible to readout the quantum number $S_{0}$ of the two outer spins by measuring the sequential tunnelling current through the central dot. The current can be calculated using the Bloch-Redfield equation approach (see App. A). For a qualitative understanding it is sufficient to consider the allowed transitions, i.e., non-zero matrix elements $\left\langle\alpha^{\prime}\left|d_{\mathrm{C}, \sigma}^{\dagger}\right| \alpha\right\rangle$, between the eigenstates of the two different charge sectors $n_{\mathrm{C}}=0$ and $n_{\mathrm{C}}=1$. The former are the eigenstates $\left|S_{0}, S_{0, z}\right\rangle$ of the total spin $\mathbf{S}_{0}=\mathbf{S}_{\mathrm{L}}+\mathbf{S}_{\mathrm{R}}$ of the two outer spins. The Hamiltonian (2) of the molecule with an additional electron can be readily diagonalized by rewriting it up to an irrelevant constant as

$$
H_{1}=-\frac{J_{\mathrm{C}}}{2} \mathbf{S}^{2}-\frac{J_{1}-J_{\mathrm{C}}}{2} \mathbf{S}_{0}^{2} .
$$

Thus, in the basis of the simultaneous eigenstates $\left|S, S_{0}, S_{z}\right\rangle$ of $\mathbf{S}^{2}, \mathbf{S}_{0}^{2}$ and $S_{z}$, the Hamiltonian in the $n_{\mathrm{C}}=1$ subsector is already diagonal. For the matrix elements $\left\langle S^{\prime}, S_{0}^{\prime}, S_{z}^{\prime}\left|d_{\mathrm{C}, \sigma}^{\dagger}\right| S_{0}, S_{0, z}\right\rangle$, we then obtain the selection rules $S^{\prime}=S_{0} \pm 1 / 2, S_{0}^{\prime}=S_{0}, S_{z}^{\prime}=S_{0, z}+\sigma$. In particular, we note that the quantum number $S_{0}$ stays 
invariant under a complete sequential-tunnelling cycle. Since furthermore the energy differences determining the tunnelling rates and thus the current depend on the quantum number $S_{0}$, we find that the (quasi-)stationary value of the current can be strongly dependent on the initial preparation of $S_{0}$.

This situation is depicted in Fig. 4, where the conductance peaks as a function of gate and bias voltage are shown schematically for two different initial values of $S_{0}$. From the figure we see that by starting initially

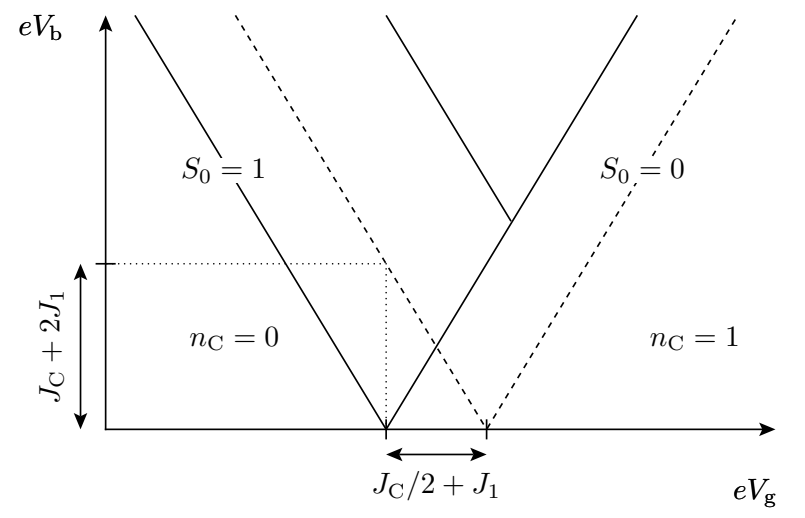

FIG. 4: Sketch of ground and excited state tunnel transitions for $S_{0}=0$ (dashed line) and $S_{0}=1$ (solid line).

at zero bias $V_{\mathrm{b}}=0$ in the $n_{\mathrm{C}}=0$ state, we first reach, upon increasing $V_{\mathrm{b}}$, the allowed ground-state transition and thus the onset of the sequential tunnelling current for the triplet $S_{0}=1$. On the other hand, for a singlet preparation $S_{0}=0$, sequential tunnelling will only be possible at a voltage which is higher by $\left(J_{\mathrm{C}}+2 J_{1}\right) / e$. At low enough tunnelling rate and temperature, i.e., $\hbar \Gamma, k_{\mathrm{B}} T \ll J_{\mathrm{C}}+2 J_{1}$, it is possible to distinguish these two cases and hence to measure the quantum number $S_{0}$. As discussed before, for an independent control of the two parameters $V_{\mathrm{g}}$ and $V_{\mathrm{b}}$ in Fig. 4, a three-terminal geometry is required. In the STM setup discussed here, one will only move along a line in Fig. 4, which, however, is sufficient for the readout scheme, if the molecule is in the $n_{\mathrm{C}}=0$ charge sector at $V_{\mathrm{b}}=0$.

Note that for an initial superposition with singlet and triplet contributions, the readout process just described represents a projective measurement. The first electron attempting to tunnel determines the final outcome, i.e., the long-time current. The average over many repeated measurements is described by the solution of the master equation A1).

We remark that the singlet-triplet readout process requires a substantially smaller tunnel coupling than the one for the exchange-controlled quantum-gate operation. There are several ways how to achieve this: Obviously, one could increase the molecule-tip distance which leads to an exponentially strong change in the tunnelling rate. Still, doing so on a very short time-scale might be technically challenging. Another possibility would be to use that the tunnel barrier-and, thus, in turn the cou- pling strength - depends on both the gate- and the biasvoltage ${ }^{26}$ Depending on the details of the contact, this might be enough to achieve the required change in the tunnel coupling. Alternatively, one could transfer the readout scheme to the so-called cotunnelling regime, $\stackrel{8,27}{=}$ where transport proceeds via virtual tunnelling on and off the molecule. Then, the onset of the inelastic cotunnelling current is solely temperature-smeared, even in the presence of a strong molecule-lead coupling. For measuring $S_{0}$ one would then need to apply a magnetic field, which will lead to a splitting of the triplet state $S_{0}=1$ only. Thus, one will find only for this state an inelastic cotunnelling step in the conductance-voltage characteristics at the bias voltage corresponding to the Zeeman energy.

\section{AB-INITIO MODELLING AND IMPLEMENTATION REQUIREMENTS}

While the preceding discussion applies to any system described by a Hamiltonian of the form (11), we now return to the specific case of the molecule $\left[\mathrm{PMo}_{12} \mathrm{O}_{40}(\mathrm{VO})_{2}\right]^{(q-)}$. A high redox flexibility is a characteristical feature of polyoxometalates, where cyclic voltammetry experiments show one- or two-electron reversible redox peaks, depending on the system and on the conditions. In particular, four compounds based on the $\mathrm{XMo}_{12} \mathrm{O}_{40}(\mathrm{X}=\mathrm{Si}, \mathrm{Ge}, \mathrm{P})$ Keggin structure (either alone or vanadyl-bicapped) have been recently found to show reversible two-electron electrochemical processes. $28 \mathrm{We}$ thus extract the exchange coupling strengths for different charge states $N$ of the molecule (see App. B). Identifying even and odd values of $N$ with $n_{\mathrm{C}}=0$ and $n_{\mathrm{C}}=1$, respectively, we obtain the parameters in the Hamiltonian (11) as given in Table I for $0 \leq N \leq 6$; for higher electronic populations $J_{0}, J_{1}$ and $J_{C}$ are of comparable size.

\begin{tabular}{|c|c|c|c|c|c|c|c|}
\hline \multicolumn{2}{|c|}{\begin{tabular}{l|l}
$N$ & $J_{0}[\mathrm{meV}$
\end{tabular}} & $E_{\text {gap }}[\mathrm{meV}]$ & & & $\underline{J_{1}}$ & & $E_{\text {gap }}$ \\
\hline 0 & 0 & $>1000$ & $N$ & $J_{\mathrm{C}}[\mathrm{meV}]$ & $\overline{J_{\mathrm{C}}}$ & $\alpha$ & $\overline{J_{\mathrm{C}}}$ \\
\hline 2 & 0.01 & $>100$ & 1 & 1 & 0.01 & $<0.1$ & $>100$ \\
\hline 4 & 0.01 & $>100$ & 3 & 1 & 0.1 & $<0.1$ & $\simeq 1$ \\
\hline 6 & 1.0 & $>100$ & 5 & 1 & 0.1 & $<0.1$ & $>10$ \\
\hline
\end{tabular}

TABLE I: Orders of magnitude of the exchange coupling strengths in the Hamiltonian (1) and estimates for the size of the correction terms, the asymmetry parameter $\alpha=\left(J_{\mathrm{CL}}-\right.$ $\left.J_{\mathrm{CR}}\right) /\left(J_{\mathrm{CL}}+J_{\mathrm{CR}}\right)$ and the gap, $E_{\text {gap }}$, between the low-energy states described by the Hamiltonian (1) and further excited states, for different electronic populations $N$. The charging energy $U$ is of the order of $1 \mathrm{eV}$.

It is worthwhile to consider how robust our predictions are against deviations from the ideal experimental setup. Specifically, (I) the orientation of the molecules may be difficult to control, (II) its electronic structure, and, specifically, its ideal symmetry, may be altered upon deposition on a surface, (III) the molecule might be affected 
by counter ions in the surrounding, and (IV) higherenergy states not described by the Hamiltonian (11) might be energetically accessible.

With respect to (I) we note that regardless of the orientation of the molecule, the change in charge state is firmly expected to affect the central $\mathrm{Mo}_{12} \mathrm{O}_{40}$ core and not the vanadyl groups, so we think orientation will only have an additional effect on (II). As an insulating thin layer separates the molecule from the metallic substrate, the alteration in the electronic structure of the polyoxometalate is expected to be minimal (details are discussed in the App. B). The main effect on the low-energy physics will be to introduce an asymmetry in the couplings between the left and right vanadyl groups and the Keggin structure, $J_{\mathrm{CL}}$ and $J_{\mathrm{CR}}$, respectively. In Table I, we give bounds for this asymmetry, which can be quantified by the parameter $\alpha=\left(J_{\mathrm{CL}}-J_{\mathrm{CR}}\right) /\left(J_{\mathrm{CL}}+J_{\mathrm{CR}}\right)$. Furthermore, we have verified that an asymmetry of up to $10 \%$ does still allow one to achieve a fidelity of $99 \%$ in the regime $J_{1} \ll J_{\mathrm{CL}}, J_{\mathrm{CR}}$ (for details see App. C). Concerning (III), cations needed to compensate the negative charge of the polyoxometalate polyanions, as well as solvent molecules, may be still present when the cluster is deposited. This feature may be a potentially important addition on (II) which could be minimized using an electrospray system to choose the mass/charge ratio corresponding to an isolated polyoxoanion. Such techniques have succesfully been applied to polyoxometalates and the peaks of the isolated anions have been detected. ${ }^{29,30}$ Finally (IV), the full diagonalization of the effective Hamiltonian also results in a set of excited states with energies $E \geq E_{\text {gap }}$ above those states described by the Hamiltonian (1): we also include these data in Table I.

Having these points in mind, we see that different charge states are usable. If we consider for instance $N=4$ and 5 , one would need molecule-lead coupling rates $\hbar \Gamma$ in the range of around 0.1 and $5 \mathrm{meV}$, for the gate and readout process, respectively. For the gate procedure, voltage pulses of a precision on the order of a few ps are required, which is not far from what can be achieved with present technology. In fact, if $N=0$ and $N=1$ are found to be chemically stable in the experimental environment, their parameters will be even more suitable for our purposes by allowing 10 times longer pulse times and yielding $J_{0}=0$ as well as $E_{\text {gap }} \gg J_{0}, J_{\mathrm{C}}$.

\section{CONCLUSIONS}

The present scheme defines a class of molecular systems: those where two localized spins are coupled to a redox-active unit. The physically relevant figures of merit are included in Table If the chemical desiderata are stability, facility of deposition on surfaces, and possibility of controlled oligomerization. Indeed, scalability of the present scheme requires covalent bonding and directed self-assembly of these logical building blocks.
Molecules of this class can be found in several chemical families, besides bicapped polyoxometalates. Tetrathiofulvalene derivatives, polypyrrol, porphyrines/phthalocyanines, fullerene and single-wall nanotubes are a few among a plethora of organic systems which can reversibly lose or gain one electron, can be substituted with radical groups, and have a rich and wellcontrolled chemistry. For example, theoretical calculations on phthalocyanines point to the possibility of the chemical tailoring of these molecules to enable exchange couplings in the range required for the gate scheme described here 31 .

While for an initial experimental realization with a single molecule, an STM contacting scheme as discussed in the present article enables the best control and is clearly favorable, a scalable method for a molecular monolayer could be based, e.g., on a crossbar architecture which already with current technology reaches very high densities 32

In conclusion, we have proposed an experimental setup for single-molecule all-electric two-qubit gate and readout which is within reach of current technology. We have exemplified our scheme using a mixed-valence polyoxometalate, for which the model parameters have been calculated using an $a b$ initio approach. The general principles behind our proposal also apply to different classes of molecular systems. The chemical design, synthesis and characterization of such systems should open new routes to molecular spin-qubit quantum computing.

\section{Acknowledgments}

We thank B. Coish for discussions. Financial support by the EU RTN QuEMolNa, the EU NoE MAGMANet, the NCCR Nanoscience, the Swiss NSF, the Spanish MEC (MAT2004-3849) and the Generalitat Valenciana is acknowledged.

\section{APPENDIX A: BLOCH-REDFIELD-EQUATION APPROACH AND AVERAGE GATE FIDELITY}

For the description of the molecular dynamics in the presence of the molecule-lead coupling we employ a Bloch-Redfield-equation formalism. This approach, which is valid in the sequential-tunnelling regime, i.e., to lowest order in the tunnelling rates $\Gamma_{\ell}$, includes the full coherent quantum dynamics of the spin states 33,34 as described by the density matrix elements $\varrho_{\alpha \beta}=$ $\operatorname{Tr}_{\text {leads }}\langle\alpha|\varrho| \beta\rangle$ after tracing out the leads. In the usual Born-Markov approximation, we obtain

$$
\begin{gathered}
\dot{\varrho}_{\alpha \beta}=-\mathrm{i} \omega_{\alpha \beta} \varrho_{\alpha \beta}+\frac{1}{2} \sum_{\ell \alpha^{\prime} \beta^{\prime}}\left\{\left[W_{\beta^{\prime} \beta \alpha \alpha^{\prime}}^{\ell}+\left(W_{\alpha^{\prime} \alpha \beta \beta^{\prime}}^{\ell}\right)^{*}\right] \varrho_{\alpha^{\prime} \beta^{\prime}}\right. \\
\left.-W_{\alpha \beta^{\prime} \beta^{\prime} \alpha^{\prime}}^{\ell} \varrho_{\alpha^{\prime} \beta}-\left(W_{\beta \alpha^{\prime} \alpha^{\prime} \beta^{\prime}}^{\ell}\right)^{*} \varrho_{\alpha \beta^{\prime}}\right\},
\end{gathered}
$$


where we have introduced the frequencies $\omega_{\alpha \beta}=\left(E_{\alpha}-\right.$ $\left.E_{\beta}\right) / \hbar$ and the transition rates $W_{\beta^{\prime} \beta \alpha \alpha^{\prime}}^{\ell}=W_{\beta^{\prime} \beta \alpha \alpha^{\prime}}^{\ell+}+$ $W_{\beta^{\prime} \beta \alpha \alpha^{\prime}}^{\ell-}$ due to tunnelling across a molecule-lead contact $\ell$. The rates for tunnelling of an electron on and

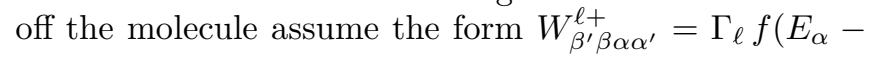
$\left.E_{\alpha^{\prime}}-\mu_{\ell}\right) \sum_{\sigma}\left\langle\beta^{\prime}\left|d_{\mathrm{C}, \sigma}\right| \beta\right\rangle\left\langle\alpha\left|d_{\mathrm{C}, \sigma}^{\dagger}\right| \alpha^{\prime}\right\rangle$ and $W_{\beta^{\prime} \beta \alpha \alpha^{\prime}}^{\ell-}=\Gamma_{\ell}[1-$ $\left.f\left(E_{\alpha^{\prime}}-E_{\alpha}-\mu_{\ell}\right)\right] \sum_{\sigma}\left\langle\beta^{\prime}\left|d_{\mathrm{C} \sigma}^{\dagger}\right| \beta\right\rangle\left\langle\alpha\left|d_{\mathrm{C}, \sigma}\right| \alpha^{\prime}\right\rangle$, respectively. Here, we assume that the lead electrons are described by a Fermi distribution $f\left(\epsilon-\mu_{\ell}\right)=\{1+\exp [(\epsilon-$ $\left.\left.\left.\mu_{\ell}\right) / k T\right]\right\}^{-1}$ at temperature $T$ and electro-chemical potential $\mu_{\ell}$. The tunnelling current across contact $\ell$ can be determined from the density matrix elements via $I_{\ell}=e \operatorname{Re} \sum_{\alpha \alpha^{\prime} \beta}\left(W_{\beta \alpha^{\prime} \alpha^{\prime} \alpha}^{\ell-}-W_{\ell \beta \alpha^{\prime} \alpha^{\prime} \alpha}^{\ell+}\right) \varrho_{\alpha \beta}$.

Using the Bloch-Redfield equation approach, we are able to simulate a realistic gate cycle. This allows us to calculate the average gate fidelity $35 \mathcal{F}=$ $\overline{\operatorname{Tr}\left[\rho_{\text {real }}\left(t_{\mathrm{f}}\right) \rho_{\text {ideal }}\right]}$ as quantitative measure for the gate quality. Here, the overline indicates the average over 16 unentangled input states $\left|\Psi_{i}\right\rangle_{\mathrm{L}}\left|\Psi_{j}\right\rangle_{\mathrm{R}}$, where $\left|\Psi_{1}\right\rangle=|\uparrow\rangle$, $\left|\Psi_{2}\right\rangle=|\downarrow\rangle,\left|\Psi_{3}\right\rangle=(|\uparrow\rangle+|\downarrow\rangle) / \sqrt{2}$, and $\left|\Psi_{4}\right\rangle=(|\uparrow\rangle+$ $\mathrm{i}|\downarrow\rangle) / \sqrt{2}, \rho_{\text {ideal }}$ is the ideal result of the $\sqrt{\text { SWAP }}$ operation as defined above and $\rho_{\text {real }}\left(t_{f}\right)$ is the state of the system according to the quantum dynamics (A1) at the final time $t_{f}$.

\section{APPENDIX B: EVALUATION OF EXCHANGE PARAMETERS}

For the evaluation of the exchange coupling constants and to check the validity of our numerical assumptions, we used an effective Hamiltonian approach. We considered the main one- and two-center phenomena in mixedvalence systems, namely magnetic exchange, electron transfer, Coulomb repulsion and orbital energy, which were parametrized for $\left[\mathrm{PMo}_{12} \mathrm{O}_{40}(\mathrm{VO})_{2}\right]^{q-}$ (details will be published elsewhere). Thus, we diagonalized a 14site $t-J-V-\epsilon$ effective Hamiltonian for different electronic populations. We projected this full energy level scheme into a subsystem containing only the localized spins in the $\left[\mathrm{V}^{I V} \mathrm{O}\right]$ groups, plus, for odd values of $N$, an unpaired spin in the $\mathrm{Mo}_{12} \mathrm{O}_{40}$ Keggin structure.

For $N$ even, $J_{0}$ is given by the energy difference between the lowest singlet and triplet states, while $E_{\text {gap }}$ is the gap to the next energy level. For $N$ odd, the evaluation of $E_{\text {gap }}$ is equally direct. Obtaining the exchange parameters is also straightforward when a symmetric molecular structure is assumed: $J_{\mathrm{C}}$ and $J_{1}$ can then be readily calculated from the energy differences between the first doublet and quadruplet states. When deviations from symmetry are considered, the determination of the three exchange parameters $J_{\mathrm{C}}, J_{\mathrm{CL}}$ and $J_{\mathrm{CR}}$ requires the analysis of the wavefunctions.

The spatially-dependent microscopic parameters $t, J$, $V$, and $\epsilon$ were obtained from ab-initio calculations of molecular subclusters, which accounts for inhomogeneities in the molecule. The experimental setup is ex- pected to imply small variations in bond distances and angles, as well as a different charge distribution in the surrounding of the molecule. In order to assess how these perturbations affect the parameters in the effective Hamiltonian (1), the calculations were repeated for a reasonable range of deviations $( \pm 50 \%$ in $J$ and $t$, and $\pm 1 \mathrm{eV}$ in $\epsilon$ ). Except for the case with $N=3$, where the excited states not contained in the Hamiltonian (1) lie close in energy, the parameters of our interest proved to be robust enough within the orders of magnitude indicated in Table 1 .

\section{APPENDIX C: CHARACTERISTIC GATE QUANTIFIERS}

Besides the average gate fidelity discussed in the main text, one can consider further quantities for assessing the quality of a quantum-gate operation ${ }^{35}$. One is the socalled gate purity

$$
\mathcal{P}=\overline{\operatorname{Tr}\left[\rho_{\text {real }}\left(t_{\mathrm{f}}\right)^{2}\right]},
$$

which is a measure for the decoherence of the gate. Here, the average is over all 16 unentangled input states $\left|\psi_{\text {ue }}\left(t_{0}\right)\right\rangle=\left|\Psi_{i}\right\rangle_{\mathrm{L}}\left|\Psi_{j}\right\rangle_{\mathrm{R}}, i, j=1, \ldots 4$ (see App. Afor the definition of the $\left.\left|\Psi_{i}\right\rangle\right)$. For an ideal $\sqrt{\text { SWAP }}$ operation it reaches unity. Another measure is the entanglement capability $\mathcal{C}$ which is defined as the smallest eigenvalue of the partial transpose of the density matrix ${ }^{36} \rho_{\text {real }}\left(t_{\mathrm{f}}\right)$ obtained for all 16 unentangled input states just defined. Ideally, it assumes a value of $-1 / 2$. Finally, the quantum degree of the gate is given by

$$
\mathcal{Q}=\max _{\left|\psi_{\text {me }}\right\rangle, \rho_{\text {ue }}\left(t_{0}\right)}\left\langle\psi_{\text {me }}\left|\rho_{\text {real }}\left(t_{\mathrm{f}}\right)\right| \psi_{\text {me }}\right\rangle,
$$

i.e., as the maximum of the overlap between all possible output states obtained for all 16 unentangled input states and all maximally entangled states $\left|\psi_{\text {me }}\right\rangle$, viz, the four Bell states $\left(|\uparrow\rangle_{L}|\uparrow\rangle_{R} \pm|\uparrow\rangle_{L}|\uparrow\rangle_{R}\right) / \sqrt{2}$ and $\left(|\downarrow\rangle_{L}|\uparrow\rangle_{R} \pm|\uparrow\rangle_{L}|\downarrow\rangle_{R}\right) / \sqrt{2}$. In the optimal case, the quantum degree reaches $1 / 2$ for the $\sqrt{\text { SWAP }}$ gate.

In Fig. [5, we show these four gate quantifiers as a function of the gating time $\tau_{\text {gate }}$ and the ratio $J_{1} /\left|J_{\mathrm{C}}\right|$. The gate fidelity $\mathcal{F}$ has already been discussed in the main text. We observe that the gate purity $\mathcal{P}$ assumes almost unity for the stroboscopic gating times (4). This means that at these times, the additional spin $\mathbf{s}_{\mathrm{C}}$ and the spin $\mathbf{S}_{0}=\mathbf{S}_{\mathrm{L}}+\mathbf{S}_{\mathrm{R}}$ become disentangled again, such that one recovers a pure state when removing the additional electron. Furthermore, we find that all gate quantifiers except for the gate fidelity overestimate the quality of the gate: They almost assume their perfect values even for parameters where the gate fidelity is much below unity. The quantum degree $\mathcal{Q}$ turns out to be completely independent of the gating time and the exchange-coupling ratio.

The situation in the presence of an asymmetry $\alpha=$ $\left(J_{\mathrm{CL}}-J_{\mathrm{CR}}\right) /\left(J_{\mathrm{CL}}+J_{\mathrm{CR}}\right)$ between the couplings $J_{\mathrm{CL}}$ and 

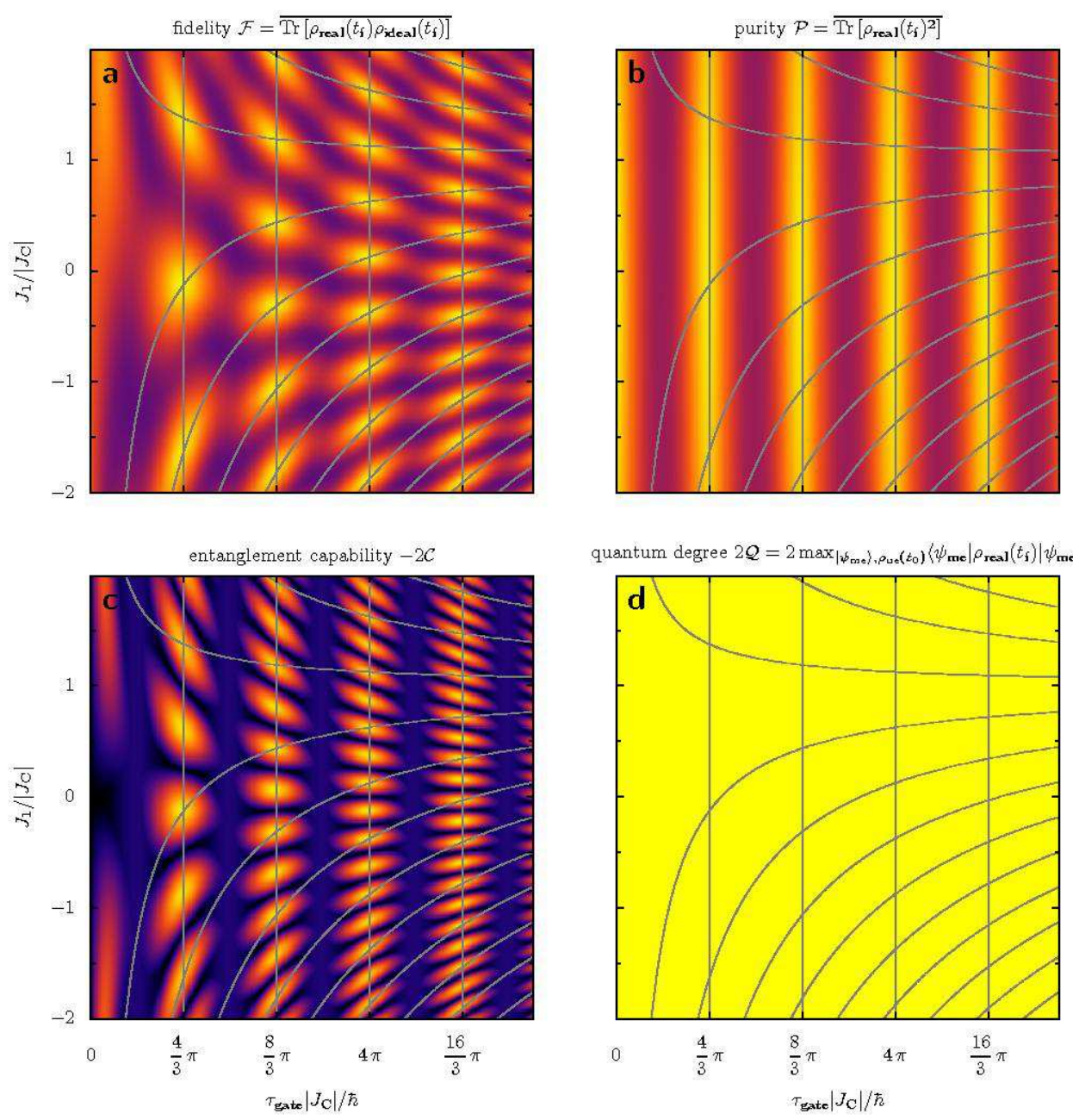

quantum degree $2 \mathcal{Q}=2 \max \left|\psi_{\text {mè }}\right\rangle$, Pue $\left(t_{0}\right)\left\langle\psi_{\text {me }}\left|\rho_{\text {real }}\left(t_{t}\right)\right| \psi_{\text {mec }}\right\rangle$
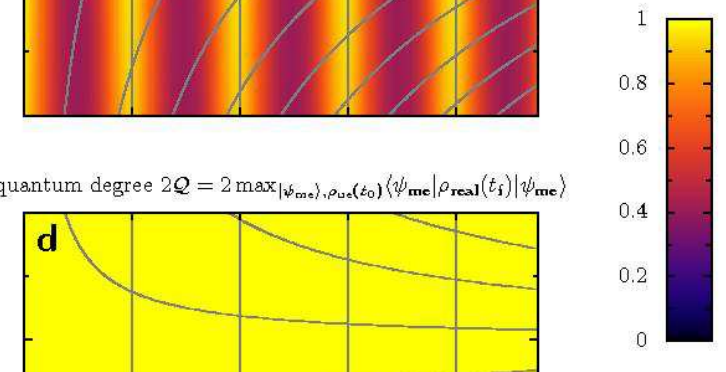

FIG. 5: Four average gate quantifiers (a) gate fidelity (b) purity (c) entanglement capability, and (d) quantum degree each normalized to the maximal value achievable for an ideal $\sqrt{\text { SWAP }}$ operation as a function of the gating time $\tau_{\text {gate }}$ and the ratio $J_{1} /\left|J_{\mathrm{C}}\right|$. The parameters are as in Fig. 3. The conditions (3) and (4) are indicated by solid lines. Note that the color scale for the gate fidelity is different from the one used in Fig. 3. 
$J_{\mathrm{CR}}$ of the central Keggin core to the left and right, respectively, vanadyl groups is shown in Fig. 6. We have chosen the upper bound $\alpha=0.1$ from the results of our $a b$ initio calculations (cf. Table I). We find for the gating times shown in Fig. 6 that this noticeable aberration from perfect symmetry does not strongly affect the gate qual- ity for small and/or negative values of the ratio $J_{1} /\left|\bar{J}_{\mathrm{C}}\right|$, where $\bar{J}_{\mathrm{C}}=\left(J_{\mathrm{CL}}+J_{\mathrm{CR}}\right) / 2$ is the average coupling of the central spin to the left and right spins. In particular, we still find a fidelity better than $\mathcal{F}=0.99$ at the maximum indicated by the circles in Fig. 6 for a tunnel rate $\Gamma=11\left|\bar{J}_{\mathrm{C}}\right| / \hbar$.
1 W. G. van der Wiel, S. De Franceschi, J. M. Elzerman, T. Fujisawa, S. Tarucha, and L. P. Kouwenhoven, Rev. Mod. Phys. 75, 1 (2003).

2 G. Burkard, D. Loss, and D. P. DiVincenzo, Phys. Rev. B 59, 2070 (1999).

3 A. Barenco, C. H. Bennett, R. Cleve, D. P. DiVincenzo, N. Margolus, P. Shor, T. Sleator, J. A. Smolin, and H. Weinfurter, Phys. Rev. A 52, 3457 (1995).

${ }^{4}$ D. Loss and D. P. DiVincenzo, Phys. Rev. A 57, 120 (1998).

5 M. A. Nielsen and I. L. Chuang, Quantum Computation and Quantum Information (Cambridge University Press, New York, 2000).

${ }^{6}$ J. R. Petta, A. C. Johnson, J. M. Taylor, E. A. Laird, A. Yacoby, M. D. Lukin, C. M. Marcus, M. P. Hanson, and A. C. Gossard, Science 309, 2180 (2005).

7 F. H. L. Koppens, C. Buizert, K. J. Tielrooij, I. T. Vink, K. C. Nowack, T. Meunier, L. P. Kouwenhoven, and L. M. K. Vandersypen, Nature 442, 766 (2006).

8 C. F. Hirjibehedin, C. P. Lutz, and A. J. Heinrich, Science 312, 1021 (2006).

9 H. B. Heersche et al., Phys. Rev. Lett. 96, 206801 (2006).

10 M.-H. Jo et al., Nano Lett. 6, 2014 (2006).

11 O. Kahn, Molecular magnetism (VCH, New York, 1993).

12 D. Gatteschi, R. Sessoli, and J. Villain, Molecular nanomagnets (Oxford University Press, Oxford, 2006).

13 W. Wernsdorfer and R. Sessoli, Science 284, 133 (1999).

14 E. Coronado, J. R. Galán-Mascarós, C. J. Gómez-García, and V. Laukhin, Nature 408, 447 (2000).

15 J. A. Real, A. B. Gaspar, V. Niel, and M. C. Muñoz, Coord. Chem. Rev. 236, 121 (2003).

16 S. Stepanow et al., Nature Materials 3, 229 (2004).

17 Hill, C. L. Special thematic issue on polyoxometalates, Chem. Rev. 98, 1-390 (1998).

18 A. Ardavan, O. Rival, J. J. L. Morton, S. J. Blundell, A. M.
Tyryshkin, G. A. Timco, and R. E. P. Winpenny, Phys. Rev. Lett. 98, 057201 (2007).

19 M. N. Leuenberger and D. Loss, Nature 410, 789 (2001).

${ }^{20}$ F. Troiani, M. Affronte, S. Carretta, P. Santini, and G. Amoretti, Phys. Rev. Lett. 94, 190501 (2005).

21 Q. Chen and C. L. Hill, Inorg. Chem. 35, 2403 (1996).

22 J. F. Keggin, Nature 131, 908 (1933).

23 S. Datta, W. Tian, S. Hong, R. Reifenberger, J. I. Henderson, and C. P. Kubiak, Phys. Rev. Lett. 79, 2530 (1997).

24 H. Park, A. K. L. Lim, A. P. Alivisatos, J. Park, and P. L. McEuen, Appl. Phys. Lett. 75, 301 (1999).

25 S. Kubatkin, A. Danilov, M. Hjort, Jérôme, C. J.-L. Brédas, N. Stuhr-Hansen, P. Hedegård, and T. Bjørnholm, Nature 425, 698 (2003).

26 E. L. Wolf, Principles of Electron Tunneling Spectroscopy (Oxford Univ. Press, New York, 1989).

27 J. Lehmann and D. Loss, Phys. Rev. B 73, 045328 (2006).

28 Z. Shi, J. Peng, C. J. Gómez-García, S. Benmansour, and X. Gu, J. Solid State Chem. 179, 253 (2006).

29 M. Bonchio, O. Bortolini, V. Conte, and A. Sartorel, Eur. J. Inorg. Chem. 4, 699 (2003).

30 C. R. Mayer, C. Roch-Marchal, H. Lavanant, R. Thouvenot, N. Sellier, J. Blais, and F. Secheresse, Chem. Eur. J. 10, 5517 (2004).

31 D. A. Shultz and K. A. Sandberg, J. Phys. Org. Chem. 12, 10 (1999).

32 J. E. Green et al., Nature 445, 414 (2007).

33 H.-A. Engel and D. Loss, Phys. Rev. Lett. 86, 4648 (2001).

34 S. Kohler, J. Lehmann, and P. Hänggi, Phys. Rep. 406, 379 (2005).

35 J. F. Poyatos, J. I. Cirac, and P. Zoller., Phys. Rev. Lett. 78, 390 (1997).

36 A. Peres, Phys. Rev. Lett. 77, 1413 (1996). 

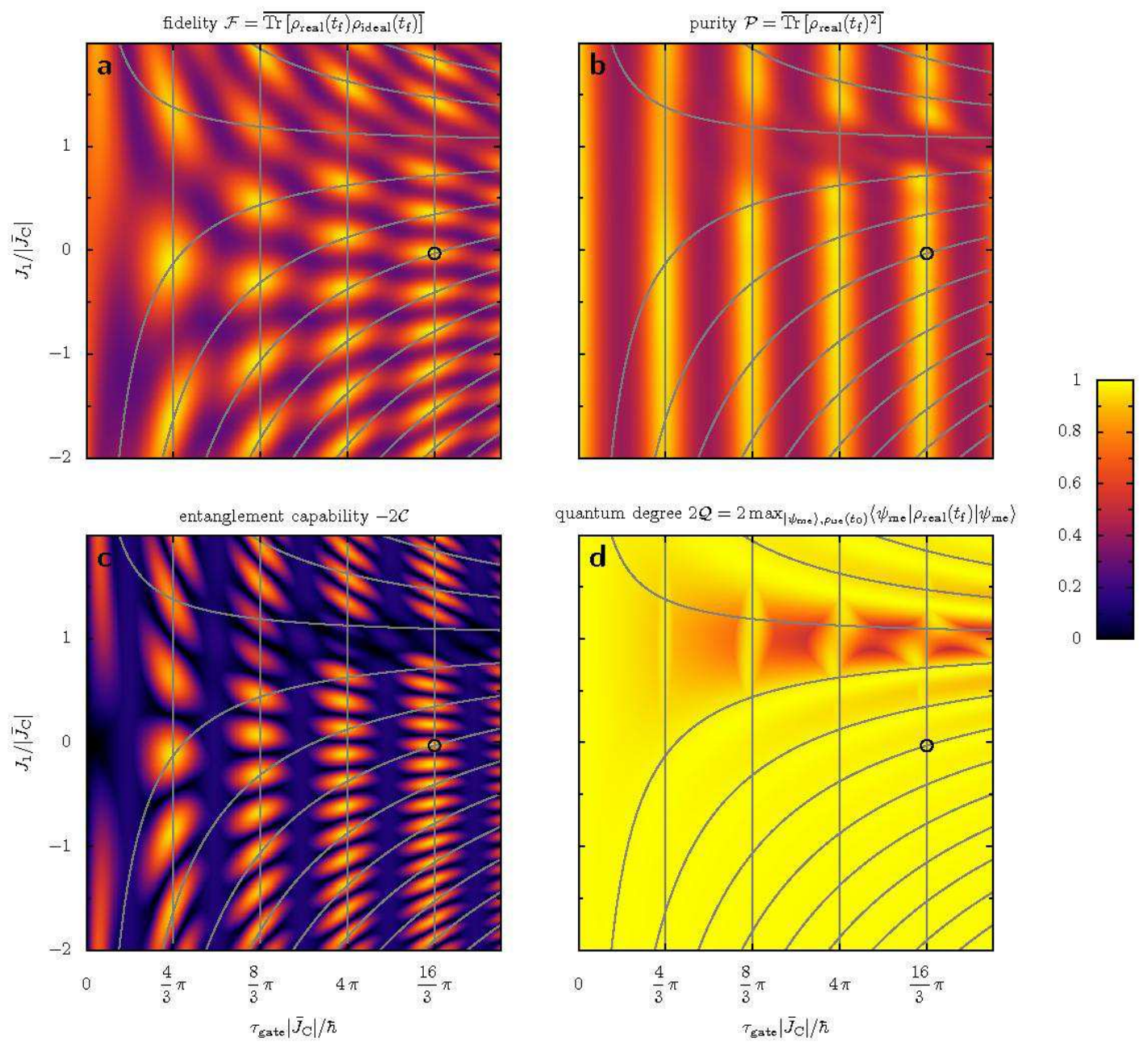

FIG. 6: Four average gate quantifiers (a) gate fidelity (b) purity (c) entanglement capability, and (d) quantum degree each normalized to the maximal value achievable for an ideal $\sqrt{\mathrm{SWAP}}$ operation as a function of the gating time $\tau_{\text {gate }}$ and the ratio $J_{1} /\left|\bar{J}_{\mathrm{C}}\right|$, where $\bar{J}_{\mathrm{C}}=\left(J_{\mathrm{CL}}+J_{\mathrm{CR}}\right) / 2$ is the average exchange coupling between the Keggin core and the left and right vanadyl groups. An asymmetry $\alpha=\left(J_{\mathrm{CL}}-J_{\mathrm{CR}}\right) /\left(J_{\mathrm{CL}}+J_{\mathrm{CR}}\right)=0.1$ of these exchange couplings is assumed. The other parameters are as in Fig. 3. The conditions (3) and (4) are indicated by solid lines. 